\title{
Two-stage laser acceleration of high quality protons using a tailored density plasma
}

\author{
Y. Wan,${ }^{1,2}$ I. A. Andriyash, ${ }^{2}$ J. F. Hua, ${ }^{1}$ C.-H. Pai, ${ }^{1}$ W. Lu, ${ }^{1, *}$ W. B. Mori, ${ }^{3}$ \\ C. Joshi, ${ }^{3}$ and V. Malka ${ }^{2,4}$ \\ ${ }^{1}$ Department of Engineering Physics, Tsinghua University, Beijing 100084, China \\ ${ }^{2}$ Department of Physics of Complex Systems, Weizmann Institute of Science, Rehovot 7610001, Israel \\ ${ }^{3}$ University of California Los Angeles, Los Angeles, California 90095, USA \\ ${ }^{4}$ Laboratoire d'Optique Appliquée, ENSTA-CNRS-Ecole Polytechnique, \\ UMR7639, 91761 Palaiseau, France
}

(Received 2 October 2018; published 5 February 2019)

\begin{abstract}
A new scheme for a laser-driven proton accelerator based on a sharply tailored near-critical-density plasma target is proposed. The designed plasma profile allows for the laser channeling of the dense plasma, which triggers a two-stage acceleration of protons-first accelerated by the laser acting as a snowplow in plasma, and then by the collisionless shock launched from the sharp density downramp. Thanks to laser channeling in the near-critical plasma, the formed shock is radially small and collimated. This allows it to generate a significant space-charge field, which acts as a monochromator, defocusing the lower energy protons while the highest ones remain collimated. Our theoretical and numerical analysis demonstrates production of high-energy proton beams with few tens of percent energy spread, few degrees divergence angle and charge up to few $\mathrm{nC}$. With a PW-class ultrashort laser this scheme predicts the generation of such high quality proton beams with energies up to several hundreds of $\mathrm{MeV}$.
\end{abstract}

DOI: 10.1103/PhysRevAccelBeams.22.021301

Over the past decades, many new exciting applications of the interactions of ultraintense lasers with matter have been developed [1]. One very attractive process is the laserdriven ion acceleration, where the ultrahigh acceleration gradients are provided by the charge-separation fields in the high density plasmas $[2,3]$. This acceleration process results in unique features of the produced beams, e.g., ultrashort durations, micron scale sizes and ultrahigh peak currents. Being very compact, such laser-based accelerators have a great potential for applications such as radiography with high spatiotemporal resolution [4-6], compact ion beam therapy of tumors $[7,8]$, etc. Many applications of accelerated ions require the beams with narrow energy spread, low divergence angle and sufficient charge.

Presently, various mechanisms of ion acceleration from laser plasmas are being explored including target-normal sheath acceleration [9], radiation pressure acceleration [10-12], breakout afterburner [13,14], laser driven shock acceleration [15-18] etc. An important role in the acceleration process is played by the target design. Among the

\footnotetext{
*weilu@tsinghua.edu.cn

Published by the American Physical Society under the terms of the Creative Commons Attribution 4.0 International license. Further distribution of this work must maintain attribution to the author(s) and the published article's title, journal citation, and DOI.
}

existing targets, of great interest are the moderate or nearcritical density (NCD) plasmas [19-24]. Relativistically intense laser can penetrate deeply into NCD plasma, and efficiently generate quasistatic accelerating electric fields. In this case, the resulting beams may contain large ion numbers $\left(\sim 10^{9}\right)$, but they typically have rather broad energy spectra, and lack angular collimation. The generation of high-charge low-divergence and narrow energyspread ion beams of several hundreds $\mathrm{MeV}$ with currently available PW-class ultrashort lasers remains a critical challenge to date.

In this article, we propose and discuss a new scheme that has the potential to generate a narrow-divergence quasimonoenergetic beam of protons that have hundreds of $\mathrm{MeV}$ energies and contain up to few $\mathrm{nC}$ charge. To achieve this, we have considered a specific plasma target, which is able to launch a strong collisionless shock with a small transverse size. The shock is needed to generate a narrow energy spectrum and a small angular divergence of accelerated protons due to a strong gate effect produced by the comoving defocusing electrostatic field of the shock itself. We show, through simulations, that the particular shock structure is achieved by using the "preparatory" stage, where laser travels through the uniform density region of the NCD plasma, expelling outwards the plasma electrons, and thus producing a plasma channel. Electrons get trapped and heated by the laser field inside the channel [25], and form the axial current trailing behind the laser. Meanwhile, 
the azimuthal magnetic field induced in the plasma channel radially confines these electrons [22]. The uniform NCD plasma is followed by a sharp density downramp, where these collimated electrons generate the required transversely narrow shock. Even before the shock formation, the laser front piles up the electrons as a "snowplow," and forms a comoving electrostatic field [26-28], which preaccelerates plasma protons in the downramp region.

This concept relies on a plasma density profile that is tailored to have a relatively long region of uniform NCD plasma followed by a sharp downramp where the density drops by a factor of a few to subcritical density. This target is irradiated by an ultrashort laser with power restricted in the present study in the range of a few hundred TW to a PW. The resulting proton beam contains a high charge, and has excellent spectral and angular quality. To develop this concept, we perform a detailed study based on threedimensional numerical simulations and support it with a theoretical model. The feasibility of this scheme is also discussed.

In the following, for convenience and model scalability, we adopt dimensionless units, normalizing time and length to $1 / \omega_{0}$ and $c / \omega_{0}$ respectively, where $\omega_{0}$ and $c$ are laser frequency and light speed in vacuum respectively. Plasma density is normalized to $n_{c}=m_{e} \omega_{0}^{2} / 4 \pi e^{2}$, and the laser field is described by the normalized amplitude $a_{0}=e E_{0} / m_{e} c \omega_{0}$, where $m_{e}, e$ are electrons rest mass and charge respectively. We set the initial time, $t_{0}=0$, to the moment when laser reaches the target front side. To model laser plasma interactions, we use the 3D relativistic particle-in-cell (PIC) code OSIRIS [29].

In our simulations, the electron-proton plasma is initially cold and fully ionized, and its density profile is shown by the gray filled curve in Fig. 1(c). The plasma starts at $200 c / \omega_{0}$ with a linear ramp $60 c / \omega_{0}$, reaching its maximum value of $n_{1}=3 n_{c}$. The entrance ramp is followed by the plateau $L_{1}=85 \mathrm{c} / \omega_{0}$, which ends with a sharp linear downramp of $d=10 c / \omega_{0}$, where density falls to $n_{2}=0.3 n_{c}$, then remains constant along the distance $L_{2}=65 c / \omega_{0}$, and finally ends with another downramp, $10 c / \omega_{0}$. We consider a circularly polarized laser focused at plasma surface at $z=$ $200 c / \omega_{0}$ with $a_{0}=42$, spot diameter (FWHM) $30 c / \omega_{0}$, and pulse duration (FWHM) $70 \omega_{0}^{-1}$. To ensure validity of the simulation, we use the numerical grid of the size of $N_{x}=N_{y}=320, N_{z}=4000$, which accurately resolves the space as $\Delta y=\Delta x=0.5 c / \omega_{0}$ and $\Delta z=0.25 c / \omega_{0}$. In simulations, plasma is initiated with 16 macroparticles per species per cell. For the reference case of a $30 \mathrm{fs}, 800 \mathrm{~nm}$ laser, the corresponding length of the plasma is about $28 \mu \mathrm{m}$, and laser power is close to $1 \mathrm{PW}$.

As mentioned above and shown in Fig. 1, in the first interaction stage, the ultraintense laser relativistically propagates through the NCD plasma at nearly luminal velocity, blowing away plasma electrons and generating a welldefined plasma channel behind. At the laser front, its

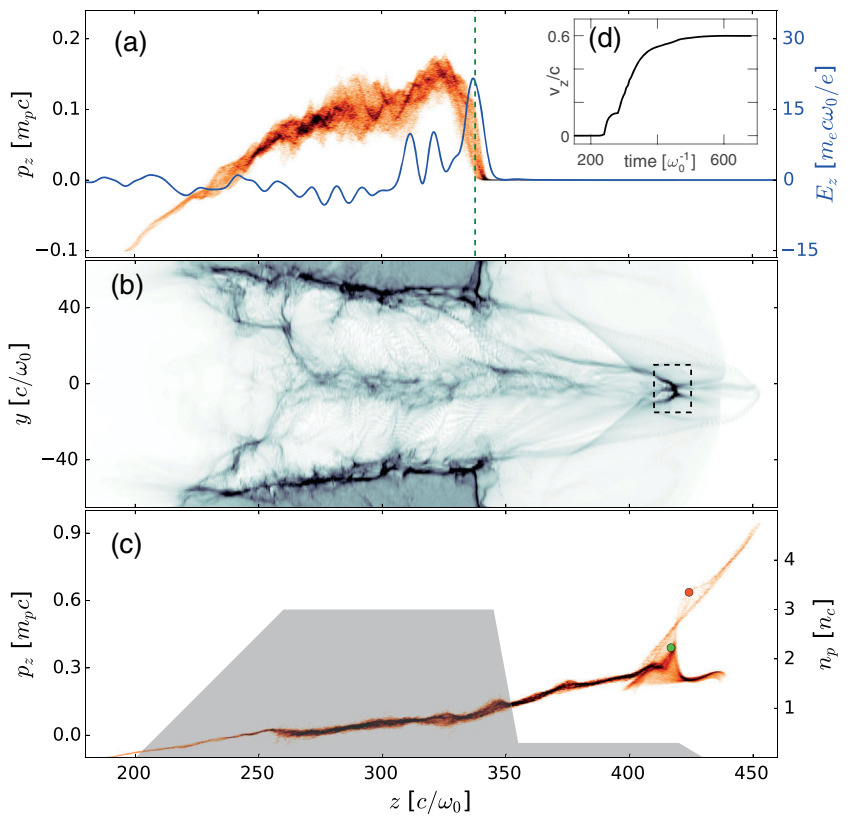

FIG. 1. (a) The averaged longitudinal electric field $E_{z}$ and proton $\left(z-P_{z}\right)$ phase space within the region $|x, y| \leq 10 c / \omega_{0}$ at $t=220 \omega_{0}^{-1}$, where the laser peak position is marked with the dashed green line. (b) The $z-y$ slice (at $x=0$ ) distribution of proton density $n_{\text {prot }}$ at $t=440 \omega_{0}^{-1}$. (c) The $\left(z-P_{z}\right)$ phase space of protons picked within the region $|x, y| \leq 10 c / \omega_{0}$, at the same time as (b). The initial plasma density profile is plotted using the gray filled curve. (d) The time evolution of a tracked proton's longitudinal velocity.

longitudinal ponderomotive force pushes plasma electrons forward, thus creating a compressed electron layer moving at relativistic velocity and often referred to as a snowplow. The charge separation between background protons and these electrons sets up a longitudinal electrostatic structure following the snowplow. As shown in Fig. 1(a), the field (blue curve) is strongest at the laser front, while afterwards it is strongly suppressed or screened by the trapped plasma electrons. This field is accelerating for positive ions, and therefore for the background protons, it turns out to be strong enough to impart a significant longitudinal momentum when the snowplow passes by [see $z-P_{z}$ phase space in Fig. 1(a)].

Inside the laser-generated channel, the induced magnetic field confines the thermal trapped plasma electrons transversely around the channel axis [22]. The effect of electron heating and collimation is enhanced in the case of circular laser polarization [30]. When passing the downramp, these electrons produce a strong pressure [31], which, together with the magnetic pressure [20,23], launches a strong shock with roughly the same small radius as the hot electron flux. In Fig. 1(b) we show the spatial structure of the shock at the later time, when it is already formed, and the laser has already left the interaction scene. The density in the shock can reach $\sim 30 n_{c}$, and its transverse size is extremely small, 


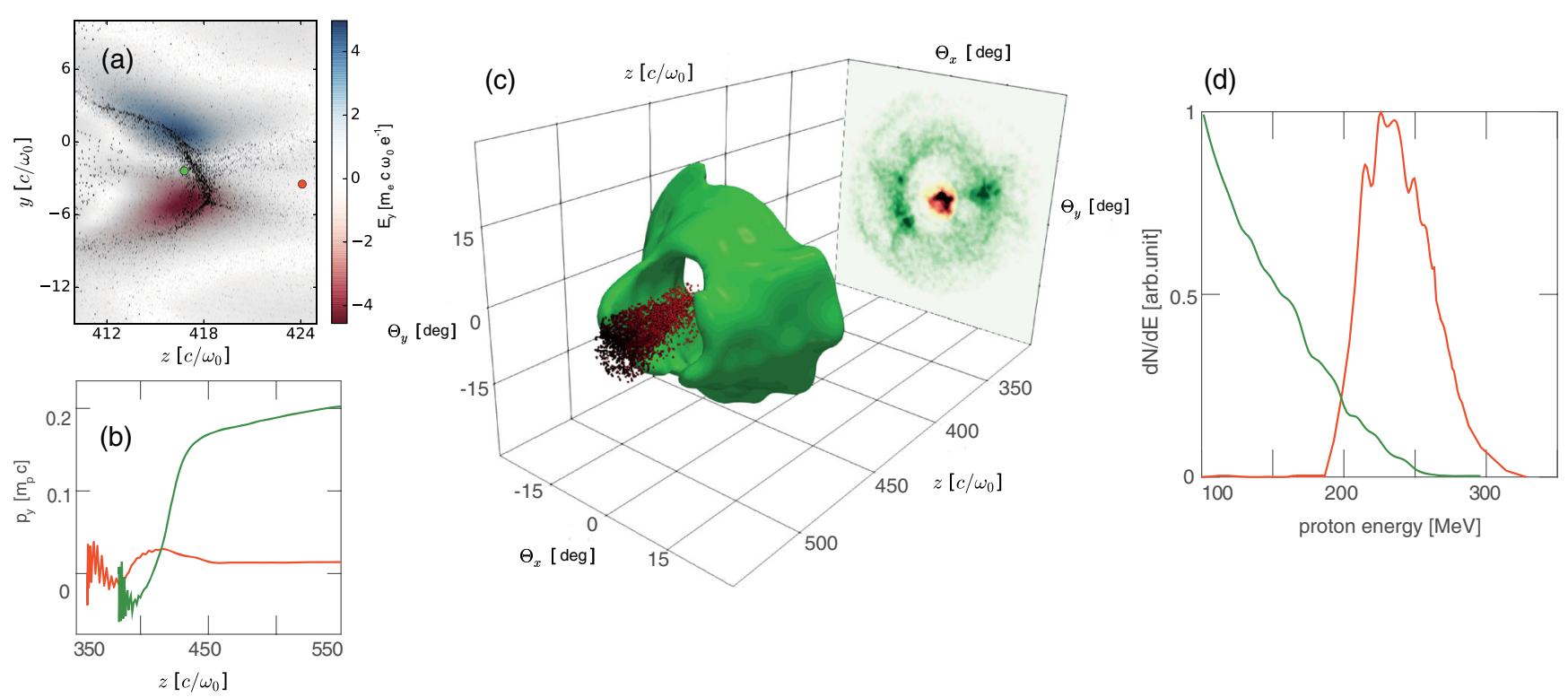

FIG. 2. (a) The $z-y$ slice (at $x=0$ ) distribution of transverse electric field $E_{y}$ and $(z-y)$ proton phase space at $t=440 \omega_{0}^{-1}$. (b) The $\left(z-P_{y}\right)$ trajectories of two sample protons traced from the positions given by the colored dots in (a) and Fig. 1(b). (c) The 3D trace space $\left(z-\theta_{x}-\theta_{y}\right)$ and its $2 \mathrm{D}$ projection $\left(\theta_{x}-\theta_{y}\right)$ for protons with the energies $\epsilon_{\text {prot }} \geq 80 \mathrm{MeV}$ at $t=600 \omega_{0}^{-1}$. (d) The energy spectra of protons (normalized individually) selected in (c). In (c) and (d), the orange and green correspond to protons with divergence angles less than $100 \mathrm{mrad}$ and more than $100 \mathrm{mrad}$, respectively.

$\sim 8 c / \omega_{0}$ (black dashed box). In Fig. 1(c), the longitudinal proton phase distribution shows that the shock velocity is $0.2 \mathrm{c}$, moving upon a upstream velocity of $0.2 \mathrm{c}$. Considering the electron temperature calculated within the shock region of about $40 m_{e} c^{2}$, the Mach number is around 1.5 , consistent with the collisionless shock theory $[31,32]$. We note that since the laser already left it does not further contribute to the acceleration process via radiation pressure (for example see [33]). As a result of such cascaded accelerations, protons obtain the maximum momentum $0.9 m_{p} c$ with a large positive chirp, where $m_{p}$ is the proton rest mass.

The two acceleration stages can be also distinguished in the time evolution of the proton velocity shown in Fig. 1(d). First the protons get accelerated in the snowplow potential during $t_{\mathrm{sp}} \sim 40 \omega_{0}^{-1}$, and reach a high velocity, $\simeq 0.15 c$. Second, the shock propagates quasi-inertially, and the protons at its front are boosted up to velocities $\simeq 0.6 c$, which corresponds to $240 \mathrm{MeV}$. Two issues need to be mentioned here. First, the snowplow contribution is significant for final proton energy. Without it, protons can only reach around $120 \mathrm{MeV}$ of energy. Second, the circular polarization of the laser enhances the capture and heating of hot electrons, thus improving the overall acceleration performance [30], so that in the linearly polarized case with the same laser energy, simulations show that the obtained proton final energy is about $150 \mathrm{MeV}$.

As mentioned before, the key to a good ion beam quality is the strong transverse electrostatic field of the radially compressed shock [see Fig. 2(a)]. The protons within or close to the shock experience strong defocusing, and acquire a significant transverse divergence, however the faster particles located in the front of the shock do not feel this field, and remain well collimated. This effect is demonstrated in Fig. 2(b) by the $\left(z-P_{y}\right)$ trajectories of two sample protons traced as colored dots in Figs. 1(c) and 2(a). One can clearly see that the proton starting ahead of the shock with higher energy (orange line) tends to move along the axis without being deflected, while the particle located closer to the shock (green line) is expelled in the transverse direction, gaining a large transverse momentum $0.2 m_{p} c$.

The described transverse defocusing process allows the separation of the high-energy and lower-divergence (collimated) protons with a quasimonoenergetic spectrum produced by the shock. The resulting transverse spectral filtering of protons is demonstrated in Fig. 2(c), where we show the proton $3 \mathrm{D}$ trace space $\left(z-\theta_{x}-\theta_{y}\right)$, and its 2D projection $\left(\theta_{x}-\theta_{y}\right)$ after exiting the plasma. For this figure, we have selected only particles with energies $\epsilon_{\text {prot }} \geq 80 \mathrm{MeV}$, and have divided them into the collimated $p_{x, y} / p_{z}<100 \mathrm{mrad}$ (orange) and divergent $p_{x, y} / p_{z}>$ $100 \mathrm{mrad}$ (green) parts. The divergent protons form a ring in the angular space, while collimated particles remain on axis. The spectra of these two groups are shown by the corresponding colors in Fig. 2(d), and we see that the collimated protons form a quasimonoenergetic beam peaked at $240 \mathrm{MeV}$ with the maximum energy close to $330 \mathrm{MeV}$, and a narrow spread (FWHM) of 20\% [orange in Fig. 2(d)]. This beam has a small divergence $\left(\simeq 1.5^{\circ}\right.$ half angle $)$, and for the case of an $800 \mathrm{~nm}$ laser, the total particle number of this 

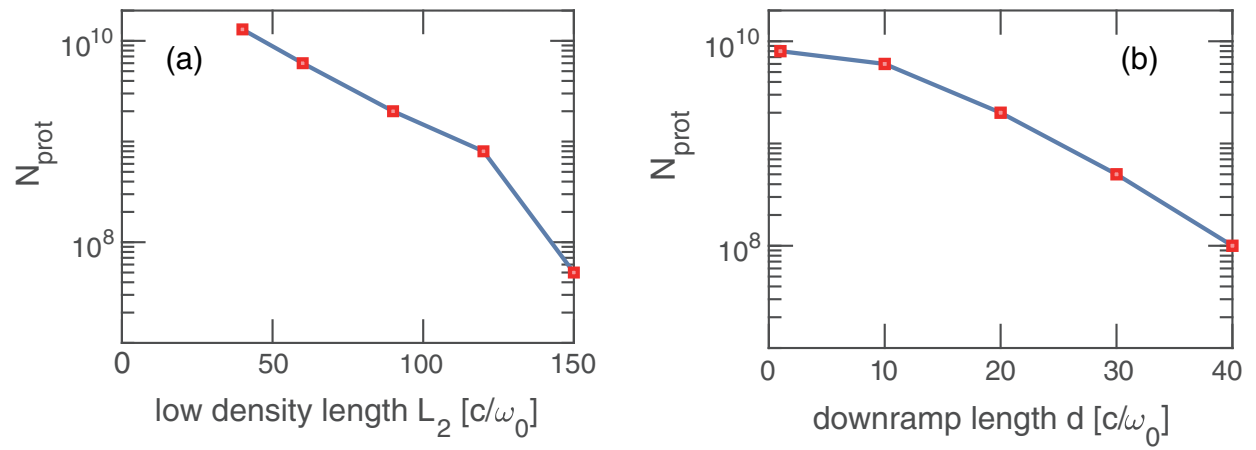

FIG. 3. The relations between numbers of collimated quasimonoenergetic protons $N_{\text {prot }}\left(\theta_{x, y}<100\right.$ mrad) and the lengths of the low density region $L_{2}$ (a) and density decreasing region $d$ (b) by fixing all other parameters.

beam estimates as $6 \times 10^{9}$. Meanwhile, the number of divergent protons in Fig. 2(c) is around $10^{11}$.

The acceleration and defocusing processes are determined by the shock dynamics. It was shown previously that the plasma Mach number for the collisionless electrostatic shock is $M_{\mathrm{sh}} \simeq 1-2$, and shock formation time is roughly $t_{\mathrm{sh}} \simeq 4 \pi / \omega_{\mathrm{pi}}$, where $\omega_{\mathrm{pi}}$ is the proton plasma frequency of high density region $[31,32]$. While it is being formed, the shock propagates a distance $L_{\mathrm{sh}}=v_{\mathrm{sh}} t_{\mathrm{sh}}$, and therefore, the plasma length of the second stage needs to be larger than this distance, $d+L_{2} \geq L_{\mathrm{sh}} \simeq 60 \mathrm{c} / \omega_{0}$. To understand better the role of plasma length, we have performed the parametric studies, by taking the total number of the collimated protons $\left(\theta_{x, y}<100 \mathrm{mrad}\right)$ as a figure of merit. In Fig. 3(a), we show the scan of $L_{2}$, which demonstrates that for the longer $L_{2}$, the total charge falls as the on-axis protons become more divergent. On the other hand, for the shorter $L_{2}$ the total charge increases, but so does the proton energy spread, and for $L_{2}=20 c / \omega_{0}$, their spectrum becomes continuous (not shown in the figure).

We find that efficiency of the shock reflection can be controlled by varying the length of the downramp plasma section. In Fig. 3(b), we confirm this by changing the downramp length for a fixed total length $d+L_{2}=L_{\mathrm{sh}}$. One can see that total quasimonoenergetic charge increases for shorter ramps, and this growth saturates for $d \lesssim 10 c / \omega_{0}$. From the performed analysis, we obtain a rough estimate of the optimal scaling [ $d \leq L_{\mathrm{sh}} / 2, L_{\mathrm{sh}} / 2 \leq L_{2} \leq 2 L_{\mathrm{sh}}$ ], which assures production of $\sim 10^{9}$ quasimonoenergetic protons.

We can derive a simple estimate of the maximal proton energy, which has a contribution from both the snowplow and shock stages. The maximal velocity achieved can be expressed as $v_{\max }=\left(v_{\mathrm{csa}}+v_{\mathrm{sp}}\right) /\left(1+v_{\mathrm{csa}} v_{\mathrm{sp}} / c^{2}\right)$, where $v_{\mathrm{csa}}=\left(2 v_{\mathrm{sh}}\right) /\left(1+v_{\mathrm{sh}}^{2} / c^{2}\right)$ is the velocity gained from the shock, and $v_{s p}$ is obtained from the snowplow potential driven by the laser. Assuming $v_{\mathrm{sp}} \ll c$, the snowplow contribution can be estimated as $v_{\text {sp }} \approx e \psi_{\text {max }} / m_{p} c$, where $\psi_{\max }$ is the maximum potential of the longitudinal electric field. For shock formation with a sharp density ramp we consider no magnetic vortex pressure $[20,23]$, and we also assume uniform shock propagation, $v_{s h}=M_{s h} c_{s}$, where $c_{s}=\sqrt{k_{B} T_{h} / m_{p}}$ is the proton acoustic velocity, $T_{h}$ is the temperature of hot plasma electrons, and $k_{B}$ is the Boltzmann constant. Expanding $v_{\max }$ as a series of $v_{\mathrm{wf}}$, we can estimate the maximum proton energy as

$\epsilon_{\max } \simeq 2 M_{\mathrm{sh}}^{2} k_{B} T_{h}+2 M_{\mathrm{sh}} e \psi_{\max } \sqrt{\frac{k_{B} T_{h}}{m_{p}}}+\frac{e^{2} \psi_{\max }^{2}}{2 m_{p}}$.

In case of a laser pulse matched to the plasma density, previous studies showed that the snowplow potential is roughly proportional to the laser ponderomotive potential, i.e., $\psi_{\max } \propto a_{0}$ for $a_{0} \gg 1$ [27]. The hot electron generation is induced by a number of phenomena including direct laser acceleration [25,30] and wave-beam instabilities [34] etc., but for a rough estimate we consider the balance between the plasma electron energy area density and the absorbed laser energy area density [35]. Assuming a uniform temperature in NCD plasma region balance equation reads $3 k_{B} T_{h} L_{1} n_{e}=\eta I \tau_{\text {laser }}$, where $I$ is laser intensity, $\tau_{\text {laser }}$ is the laser pulse duration, and $\eta$ is the laser to electron conversion efficiency. Therefore, knowing the laser absorption efficiency $\eta$, and coefficient between $\psi_{\max }$ and $a_{0}$, one can readily get the maximum proton energy.

In order to verify this scaling, and to find the proper coefficients, we have performed another parametric study. In this study we have scaled up the laser intensity and plasma densities, but fixed the laser and plasma spatial scales (laser spot size/duration, plasma $L$ and $d$ lengths) to be the same as the simulation shown in Figs. 1 and 2. We have scanned $a_{0}$ from 9 to 55, at the same time increasing $n_{1}$ proportionally from $0.6 n_{c}$ to $4 n_{c}$, to maintain the matched spot size. The ratio $n_{1} / n_{2}$ was chosen as 10 , for effective shock formation [31]. From these simulations we could estimate the coupling efficiency $\eta=30 \%$ $\left(k_{B} T_{h} / m_{e} c^{2} \sim a_{0}\right)$, and $e \psi_{\max } / m_{e} c^{2} \sim 4 a_{0}$. In Fig. 4(a) we demonstrate the proton energy spectra for the different $a_{0}$, which shows that the quasimonoenergetic feature is preserved reasonably along the scaling. 

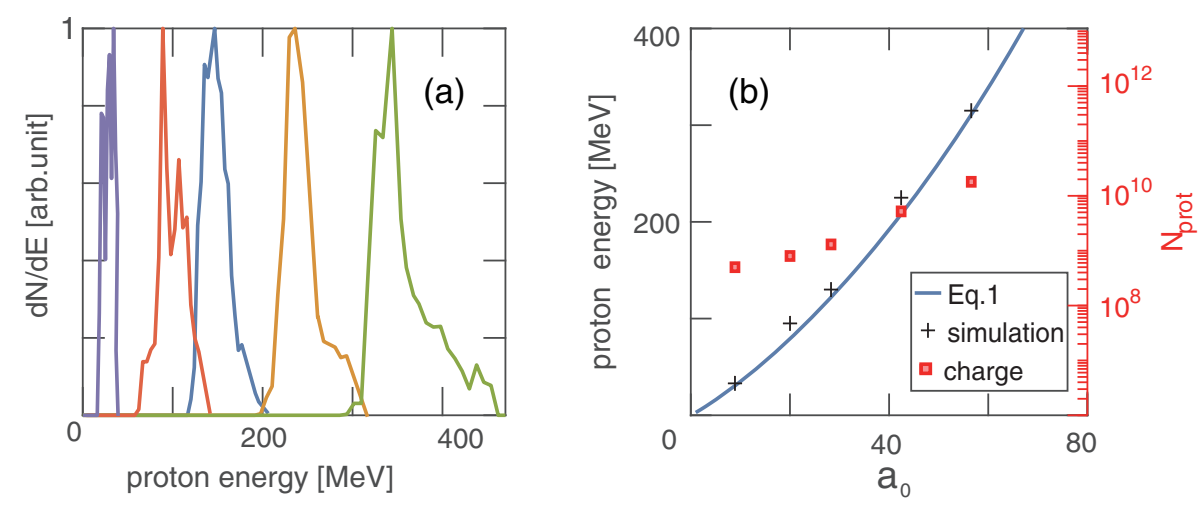

FIG. 4. (a) Spectrum of proton beams for different laser plasma parameters corresponding to $a_{0}=9$ (purple), 20 (red), 28 (blue), 42 (orange) and 55 (green). (b) The relation between proton peak energy $\epsilon_{\max }$ and particle numbers $N_{\text {prot }}$ with laser $a_{0}$.

The scaling for the proton peak energy is plotted in Fig. 4 (b), and it agrees well with the theory Eq. (1), which indicates that at low laser intensities (e.g., $a_{0}=9$ ) acceleration is mainly dominated by shock acceleration [first term of Eq. (1)] and scales as $\epsilon_{\max } \propto a_{0}$, while for higher intensities, the snowplow contribution becomes significant [second and third terms of Eq. (1)], which improves the scaling to $\propto a_{0}^{3 / 2}$ and further to $\propto a_{0}^{2}$. The particle numbers in all cases are around $10^{9}-10^{10}$, and their divergence angles decrease with the increased laser power from $3^{\circ}$ to $1.2^{\circ}$. The conversion efficiency is around $1 \%$.

The proposed scheme can be readily tested with ultraintense ultrashort lasers. Our model predicts that high quality proton beams with energies from 100 to $300 \mathrm{MeV}$ can be obtained using laser powers from $200 \mathrm{TW}$ to $1 \mathrm{PW}$ $\left(a_{0}=20-42\right)$. One option for the driver can be a commercially available Ti:sapphire laser system with $\lambda_{0}=800 \mathrm{~nm}$ wavelength, which requires a few tens of microns long NCD plasma $\left(n_{p} \sim 10^{20}-10^{21} \mathrm{~cm}^{-3}\right)$ with a downramp of a few micrometers. Such a target can be fabricated using a high pressure gas jet [36], with a density ramp formed by laser machining of the plasma. For this machining a low-power laser is used to preheat plasma locally, thus creating smallscale features in its density profile [37]. Another option can be to use a $\mathrm{CO}_{2}$ laser system with $\lambda_{0}=10 \mu \mathrm{m}$, for which $\mathrm{NCD}$ can be achieved with gas densities of $\sim 10^{19} \mathrm{~cm}^{-3}$ [16]. The next generations of $\mathrm{CO}_{2}$ lasers promise to deliver short $100 \mathrm{TW}$ pulses [38] that are capable of high quality beams of $100 \mathrm{MeV}$-level protons. It is noted that such a NCD gas target is much less demanding on laser contrast, where low energy prepulses are diffracted before reaching the sharp downramp region.

In summary, we have presented a new efficient laser plasma acceleration scheme for production of quasimonoenergetic proton beams. In the proposed scheme, a sharply tailored NCD plasma target is employed to enable the laser to propagate through, thus launching a strong electrostatic snowplow potential and producing a limitedsize dense plasma shock. We show that this scenario leads to the beam self-cleaning through the defocusing process, so that only a high energy, narrow spectrum part of the beam remains collimated. Practically, this allows compact production of the application-ready proton beams without complicity of such conventional beam transport techniques as magnetic collimators and monochromators. Another distinctive advantage of this scheme is the favorable scalability with laser energy, which makes it efficient on the lasers of different classes-from the commercially available 100 TW lasers to the state-of-the-art multi-PW systems.

This work was supported by NSFC Grants No. 11425521, No. 11535006, No. 11475101 and No. 11775125, the Thousand Young Talents Program, DOE Grant No. DE-NA0003842. Simulations were performed on Sunway TaihuLight cluster at National Supercomputing Center and Edison cluster at NERSC.

[1] G. A. Mourou, T. Tajima, and S. V. Bulanov, Optics in the relativistic regime, Rev. Mod. Phys. 78, 309 (2006).

[2] H. Daido, M. Nishiuchi, and A. S. Pirozhkov, Review of laser-driven ion sources and their applications, Rep. Prog. Phys. 75, 056401 (2012).

[3] A. Macchi, M. Borghesi, and M. Passoni, Ion acceleration by superintense laser-plasma interaction, Rev. Mod. Phys. 85, 751 (2013).

[4] M. Borghesi, S. Bulanov, D. H. Campbell, R. J. Clarke, T. Z. Esirkepov, M. Galimberti, L. A. Gizzi, A. J. MacKinnon, N. M. Naumova, F. Pegoraro, H. Ruhl, A. Schiavi, and O. Willi, Macroscopic Evidence of Soliton Formation in Multiterawatt Laser-Plasma Interaction, Phys. Rev. Lett. 88, 135002 (2002).

[5] A. J. Mackinnon et al., Proton Radiography of a LaserDriven Implosion, Phys. Rev. Lett. 97, 045001 (2006).

[6] C. K. Li, F. H. Seguin, J. A. Frenje, J. R. Rygg, R. D. Petrasso, R. P. J. Town, P. A. Amendt, S. P. Hatchett, O. L. Landen, A. J. Mackinnon, P. K. Patel, V. A. Smalyuk, T. C. Sangster, and J. P. Knauer, Measuring $E$ and $B$ Fields in Laser-Produced Plasmas with Monoenergetic Proton Radiography, Phys. Rev. Lett. 97, 135003 (2006). 
[7] S. V. Bulanov, T. Z. Esirkepov, V. S. Khoroshkov, A. V. Kunetsov, and F. Pegoraro, Oncological hadrontherapy with laser ion accelerators, Phys. Lett. A 299, 240 (2002).

[8] V. Malka, S. Fritzler, E. Lefebvre, E. d'Humieres, R. Ferrand, G. Grillon, C. Albaret, S. Meyroneinc, J. P. Chambaret, A. Antonetti, and D. Hulin, Practicability of protontherapy using compact laser systems, Med. Phys. 31, 1587 (2004).

[9] R. A. Snavely, M. H. Key, S. P. Hatchett, T. E. Cowan, M. Roth, T. W. Phillips, M. A. Stoyer, E. A. Henry, T. C. Sangster, M. S. Singh, S. C. Wilks, A. MacKinnon, A. Offenberger, D. M. Pennington, K. Yasuike, A. B. Langdon, B. F. Lasinski, J. Johnson, M. D. Perry, and E. M. Campbell, Intense High-Energy Proton Beams from Petawatt-Laser Irradiation of Solids, Phys. Rev. Lett. 85, 2945 (2000).

[10] T. Esirkepov, M. Borghesi, S. V. Bulanov, G. Mourou, and T. Tajima, Highly Efficient Relativistic-Ion Generation in the Laser-Piston Regime, Phys. Rev. Lett. 92, 175003 (2004).

[11] A. Macchi, F. Cattani, T. V. Liseykina, and F. Cornolti, Laser Acceleration of Ion Bunches at the Front Surface of Overdense Plasmas, Phys. Rev. Lett. 94, 165003 (2005).

[12] Y. Wan, C. H. Pai, C. J. Zhang, F. Li, Y. P. Wu, J. F. Hua, W. Lu, Y. Q. Gu, L. O. Silva, C. Joshi, and W. B. Mori, Physical Mechanism of the Transverse Instability in Radiation Pressure Ion Acceleration, Phys. Rev. Lett. 117, 234801 (2016).

[13] B. J. Albright, L. Yin, K. J. Bowers, B. M. Hegelich, K. A. Flippo, T. J. T. Kwan, and J. C. Fernandez, Relativistic Buneman instability in the laser breakout afterburner, Phys. Plasmas 14, 094502 (2007).

[14] L. Yin, B. J. Albright, B. M. Hegelich, K. J. Bowers, K. A. Flippo, T. J. T. Kwan, and J. C. Fernandez, Monoenergetic and $\mathrm{GeV}$ ion acceleration from the laser breakout afterburner using ultrathin targets, Phys. Plasmas 14, 056706 (2007).

[15] L. O. Silva, M. Marti, J. R. Davies, R. A. Fonseca, C. Ren, F. S. Tsung, and W. B. Mori, Proton Shock Acceleration in Laser-Plasma Interactions, Phys. Rev. Lett. 92, 015002 (2004).

[16] D. Haberberger, S. Tochitsky, F. Fiuza, C. Gong, R. A. Fonseca, L. O. Silva, W. B. Mori, and C. Joshi, Collisionless shocks in laser-produced plasma generate monoenergetic high-energy proton beams, Nat. Phys. 8, 95 (2012).

[17] F. Fiuza, A. Stockem, E. Boella, R. A. Fonseca, L. O. Silva, D. Haberberger, S. Tochitsky, C. Gong, W. B. Mori, and C. Joshi, Laser-Driven Shock Acceleration of Monoenergetic Ion Beams, Phys. Rev. Lett. 109, 215001 (2012).

[18] A. Pak, S. Kerr, N. Lemos, A. Link, P. Patel, F. Albert, L. Divol, B. Pollock, D. Haberberger, D. Froula et al., Collisionless shock acceleration of narrow energy spread ion beams from mixed species plasmas using $1 \mu \mathrm{m}$ lasers, Phys. Rev. Accel. Beams 21, 103401 (2018).

[19] A. V. Kuznetsov, T. Z. Esirkepov, F. F. Kamenets, and S. V. Bulanov, Efficiency of ion acceleration by a relativistically strong laser pulse in an underdense plasma, Plasma Phys. Rep. 27, 211 (2001).

[20] S. V. Bulanov, D. V. Dylov, T. Z. Esirkepov, F. F. Kamenets, and D. V. Sokolov, Ion acceleration in a dipole vortex in a laser plasma corona, Plasma Phys. Rep. 31, 369 (2005).
[21] L. Willingale, S. P. D. Mangles, P. M. Nilson, R. J. Clarke, A. E. Dangor, M. C. Kaluza, S. Karsch, K. L. Lancaster, W. B. Mori, Z. Najmudin, J. Schreiber, A. G. R. Thomas, M. S. Wei, and K. Krushelnick, Collimated Multi-MeV Ion Beams from High-Intensity Laser Interactions with Underdense Plasma, Phys. Rev. Lett. 96, 245002 (2006).

[22] S. S. Bulanov, V. Y. Bychenkov, V. Chvykov, G. Kalinchenko, D. W. Litzenberg, T. Matsuoka, A. G. R. Thomas, L. Willingale, V. Yanovsky, K. Krushelnick, and A. Maksimchuk, Generation of $\mathrm{GeV}$ protons from $1 \mathrm{PW}$ laser interaction with near critical density targets, Phys. Plasmas 17, 043105 (2010).

[23] T. Nakamura, S. V. Bulanov, T.Z. Esirkepov, and M. Kando, High-Energy Ions from Near-Critical Density Plasmas via Magnetic Vortex Acceleration, Phys. Rev. Lett. 105, 135002 (2010).

[24] M. H. Helle, D. F. Gordon, D. Kaganovich, Y. Chen, J. P. Palastro, and A. Ting, Laser-Accelerated Ions from a ShockCompressed Gas Foil, Phys. Rev. Lett. 117, 165001 (2016).

[25] A. Pukhov, Z. M. Sheng, and J. M. ter Vehn, Particle acceleration in relativistic laser channels, Phys. Plasmas 6, 2847 (1999).

[26] L. O. Silva, R. Bingham, J. M. Dawson, and W. B. Mori, Ponderomotive force of quasiparticles in a plasma, Phys. Rev. E 59, 2273 (1999).

[27] A. A. Sahai, F. S. Tsung, A. R. Tableman, W. B. Mori, and T. C. Katsouleas, Relativistically induced transparency acceleration of light ions by an ultrashort laser pulse interacting with a heavy-ion-plasma density gradient, Phys. Rev. E 88, 043105 (2013).

[28] H. Wang, X. Yan, and M. Zepf, High-energy monoenergetic proton beams from two stage acceleration with a slow laser pulse, Phys. Rev. ST Accel. Beams 18, 021302 (2015).

[29] R. A. Fonseca, L. O. Silva, F. S. Tsung, V. K. Decyk, W. Lu, C. Ren, W. B. Mori, S. Deng, S. Lee, T. Katsouleas, and J.C. Adam, OSIRIS: A three-dimensional, fully relativistic particle in cell code for modeling plasma based accelerators, Lect. Notes Comput. Sci. 2331, 342 (2002).

[30] B. Liu, H. Y. Wang, J. Liu, L. B. Fu, Y. J. Xu, X. Q. Yan, and X. T. He, Generating Overcritical Dense Relativistic Electron Beams via Self-Matching Resonance Acceleration, Phys. Rev. Lett. 110, 045002 (2013).

[31] F. Fiuza, A. Stockem, E. Boella, R. A. Fonseca, L. O. Silva, D. Haberberger, S. Tochitsky, W. B. Mori, and C. Joshi, Ion acceleration from laser-driven electrostatic shocks, Phys. Plasmas 20, 056304 (2013).

[32] D. W. Forslund and C. R. Shonk, Formation and Structure of Electrostatic Collisionless Shocks, Phys. Rev. Lett. 25, 1699 (1970).

[33] L. Ji, A. Pukhov, and B. Shen, Ion acceleration in the 'dragging field' of a light-pressure-driven piston, New J. Phys. 16, 063047 (2014).

[34] A. Debayle, F. Mollica, B. Vauzour, Y. Wan, A. Flacco, V. Malka, X. Davoine, and L. Gremillet, Electron heating by intense short-pulse lasers propagating through near-critical plasmas, New J. Phys. 19, 123013 (2017).

[35] Y. Wan, C.-H. Pai, J. Hua, Y. Wu, W. Lu, F. Li, C. Zhang, $\mathrm{X}$. Xu, C. Joshi, and W. Mori, Tri-stage quasimonoenergetic proton acceleration from a multi-species thick target, Phys. Plasmas 25, 073105 (2018). 
[36] M. Sylla, M. Veltcheva, S. Kahaly, A. Flacco, and V. Malka, Development and characterization of very dense submillimetric gas jets for laser-plasma interaction, Rev. Sci. Instrum. 83, 033507 (2012).

[37] C. H. Pai, S. Y. Huang, C. C. Kuo, M. W. Lin, J. Wang, S. Y. Chen, C. H. Lee, and J. Y. Lin, Fabrication of spatial transient-density structures as high-field plasma photonic devices, Phys. Plasmas 12, 070707 (2005).

[38] I. V. Pogorelsky and I. Ben-Zvi, Brookhaven National Laboratory's Accelerator Test Facility: research highlights and plans, Plasma Phys. Controlled Fusion 56, 084017 (2014). 